\title{
Time-triggered control of nonlinear discrete-time systems
}

\author{
Romain Postoyan and Dragan Nešić
}

\begin{abstract}
We investigate the time-triggered control of nonlinear discrete-time systems using an emulation approach. We assume that we know a controller, which stabilizes the origin of a discrete-time nonlinear system. We then provide conditions to preserve stability when the control input is no longer updated at each step, but within $N$ steps from the previous update, where $N$ is a strictly positive integer. We consider general output feedback controllers and we allow for various holding strategies of the control input between two updates, such as zero-input or hold-input policies for example. An easily computable bound on the maximum number of steps between two updates, i.e. $N$, is provided. The results are applied to linear time-invariant systems in which case the assumptions are written as a linear matrix inequality, and a nonlinear physical example is provided as an illustration. This study is relevant for networked control systems, as well as any system for which sparse or sporadically changing control inputs are advisable in view of the resource limitations for instance.
\end{abstract}

\section{INTRODUCTION}

Control strategies that generate sparse or sporadically varying inputs are suitable in a number of situations. Networked control systems (NCS) are a typical example. These are systems in which the plant and the controller communicate with each other via a digital channel, which may be used by other tasks. In this set-up, the frequent transmission of data between the plant and the controller, and thus the frequent update of the control input, may exceed the communication channel capacity, and leads to delays or packet losses, which may destroy the desired properties of the closed-loop system. Another example is embedded systems, for which the computation of the control input is limited by the available computation resources. Similar situations arise in a variety of other control applications. In medicine for instance, more specifically in dynamic phototherapy [7], to develop sparse control policies would be beneficial for the patients in order to reduce the pain induced by the treatment [1].

Several types of such control strategies are available in the literature. The simplest option is to hold the control input for a fixed amount of time, leading to time-triggered control, see e.g., [6], [18]. An alternative consists in adapting the transmissions according to the current state of the plant, we talk of event-triggered control, see [10], [22] and the references

R. Postoyan is with the Université de Lorraine, CRAN, UMR 7039 and the CNRS, CRAN, UMR 7039, France romain.postoyan@univ-lorraine.fr. His work was partially supported by the ANR under the grant COMPACS (ANR-13-BS03-0004$02)$.

D. Nešić is with the Department of Electrical and Electronic Engineering, the University of Melbourne, Parkville, VIC 3010, Australia, dnesiceunimelb.edu.au. His work was supported by the Australian Research Council under the Discovery Project DP1094326. therein. The idea is to evaluate a state-dependent criterion at each step, whenever the latter is satisfied, a transmission is triggered. Event-triggered control usually generates less transmissions than time-triggered control, however the evaluation of the transmission condition may be difficult to implement in some applications. In self-triggered control, transmissions also adapt to the system evolution but the next triggering instant is decided based on the value of the state at the last control input update (and not the current state as in event-triggered control, to avoid the frequent evaluation of the triggering condition), see [10] and the references therein. The choice between these paradigms depends on the considered problem, all are relevant and deserve being investigated.

We study nonlinear discrete-time systems in this paper. This is justified by the fact that many processes are conveniently modeled in discrete-time because of their intrinsic nature or because of the tools used to construct the model, see [15] for instance. Also, any sufficiently smooth continuoustime model can be (approximately) discretized. Results, which relate the stability of nonlinear discretized models to the stability of the original system can be found in [19]; we do not address this issue in this paper and leaves it for future work. In the context of NCS, to work with a discrete model may be convenient as it allows considering holding strategies in which the input is set to zero when no transmission has occurred to reduce the actuation cost, see [24] for instance. Furthermore, equidistant transmissions naturally give rise to discrete-time models of the form considered here. Results on the event-triggered control of discrete-time systems can be found in [5], [8], [13] for instance. Recently, many works have been devoted to the design of predictive strategies for NCS, see e.g., [2], [4], [11], [23]. At each triggering instant, a packet containing future values of the control input is sent to the plant, and then stored in a buffer until the next transmission occurs. Hence, in [3], time-triggered and self-triggered policies are presented for general nonlinear discrete-time systems in order to optimize a discounted cost. While this strategy allows reducing the usage of the network, it still updates the control input value at each iteration. To overcome this potential issue, self-triggered controllers for constrained nonlinear systems are proposed in [9], which generate sparse or sporadically changing control inputs, depending on the desired specification, while guaranteeing stability and a desired level of optimality.

In this study, we focus on time-triggered control and our objective is to address the following problem. We assume that we know an output-feedback controller, which uniformly asymptotically stabilizes the origin of the plant when it is 
updated at each step. We then consider the scenario where a new control input is transmitted to the plant within every $N$ steps from the last transmission. Between two updates, the input is held using a general type of functions, which captures the hold-input and the zero-input [24] policies as particular cases. We provide conditions on the closedloop system as well as an explicit bound on the maximum allowable transmission interval (MATI), i.e. $N$, to guarantee stability. In particular, sufficient conditions for local/global, asymptotic/exponential stability are given. These conditions are written as a linear matrix inequality for linear timevarying systems, and a discretized Lorenz model of a thermal convection loop is proved to satisfy the required assumptions.

The approach is inspired by the continuous-time results in [18]. It appears that the discrete-time nature of the problem generates differences and some non-trivial technical difficulties compared to [18]. Indeed, we first had to modify the assumptions on the closed-loop system, and we had to make an assumption on a $\ell_{2}$-gain contrary to [18], as will be explained later. We then provide a bound on the MATI, which is different from the one in continuous-time. Indeed, in contrast with [18], we do not provide an explicit formula for the MATI bound but a simple algorithm for its computation, which is easy to implement. Compared to the related works based on model predictive ideas [3], [9], the controller is an output feedback law (we do not require to measure the full state) and we do not rely on optimization techniques, which may be important for applications with low computational capacities.

\section{Preliminaries}

Let $\mathbb{R}:=(-\infty, \infty), \mathbb{R}_{\geq 0}:=[0, \infty)$, and $\mathbb{Z}_{\geq 0}:=$ $\{0,1,2, \ldots\}$. For $(x, y) \in \mathbb{R}^{n+m},(x, y)$ stands for $\left[x^{\mathrm{T}}, y^{\mathrm{T}}\right]^{\mathrm{T}}$. A function $\chi: \mathbb{R}_{\geq 0} \rightarrow \mathbb{R}_{\geq 0}$ is of class $\mathcal{K}$ if it is continuous, zero at zero and strictly increasing, and it is of class $\mathcal{K}_{\infty}$ if, in addition, it is unbounded. A continuous function $\chi: \mathbb{R}_{>0}^{2} \longrightarrow \mathbb{R}_{\geq 0}$ is of class $\mathcal{K} \mathcal{L}$ if for each $t \in \mathbb{R}_{\geq 0}, \chi(\cdot, t)$ is of class $\mathcal{K}$, and, for each $s>0, \chi(s, \cdot)$ is decreasing to zero. The distance of a point $x \in \mathbb{R}^{n}$ to a set $\mathcal{A} \subseteq \mathbb{R}^{n}$ is denoted by $|x|_{\mathcal{A}}:=\inf \{|x-y|: y \in \mathcal{A}\}$. Let $P$ be a real, square, and symmetric matrix, $\lambda_{\max }(P)$ and $\lambda_{\min }(P)$ are respectively the largest and the smallest eigenvalue of $P$. The notation $\mathbb{I}$ stands for the identity matrix. The symbol $\star$ used in matrices denotes the symmetric block component.

We will use the following stability definition.

Definition 1: Consider the system $x^{+}=F(x)$ with $x \in$ $\mathbb{R}^{n}$. The compact set $\mathcal{S} \subset \mathbb{R}^{n}$ is:

- uniformly locally asymptotically stable (ULAS) if there exist $\beta \in \mathcal{K} \mathcal{L}$ and $c>0$, such that for any $x(0)$ with $|x(0)|_{\mathcal{S}} \leq c$, the corresponding solution verifies $|x(k)|_{\mathcal{S}} \leq \beta\left(|x(0)|_{\mathcal{S}}, k\right)$ for any $k \in \mathbb{Z}_{\geq 0}$.

- uniformly globally asymptotically stable (UGAS) if the previous property holds for any $x(0)$.

- uniformly locally (respectively, globally) exponentially stable (ULES (respectively, UGES)) if it is ULAS (respectively, UGAS) and $\beta(s, k)=C e^{-\sigma k}$ with $C>0$ and $\sigma>0$ for any $s, k \in \mathbb{Z}_{\geq 0}$.

\section{Problem statement}

Consider the system

$$
x_{p}^{+}=f_{p}\left(x_{p}, u\right), \quad y=g_{p}\left(x_{p}\right),
$$

with state $x_{p} \in \mathbb{R}^{n_{p}}$, input $u \in \mathbb{R}^{n_{u}}$, and output $y \in \mathbb{R}^{n_{y}}$. We design the following controller to stabilize system (1)

$$
x_{c}^{+}=f_{c}\left(x_{c}, y\right), \quad u=g_{c}\left(x_{c}, y\right),
$$

where $x_{c} \in \mathbb{R}^{n_{c}}$ is the controller state. System (2) covers static controllers, in which case $u=g_{c}(y)$ (and no variable $x_{c}$ is needed).

We study the scenario where the input to system (1) is no longer updated by (2) at each step but sporadically, such that there exists a maximum of $N$ steps between two successive updates. The underlying idea is to transmit as rarely as possible. Hence, denoting $k_{i}, i \in \mathbb{Z}_{>0}$, the sequence of transmission instants, $k_{i+1}-k_{i} \leq N$ for any $i \in \mathbb{Z}_{\geq 0}$. At each $k_{i}$, the controller receives the current value of the plant output $y$, generates an updated control input $u$, which is immediately transmitted to the plant. We assume that the delays induced by the transmissions and the computation time are negligible. For the sake of convenience, we introduce the clock variable $\tau \in \mathbb{Z}_{\geq 0}$ to count the number of steps since the last transmission. Variable $\tau$ has the following dynamics

$$
\tau^{+}=\left\{\begin{array}{lrr}
1 & \text { when } \quad \tau \in\{1, \ldots, N\} \\
& & \text { (transmission) } \\
\tau+1 & \text { when } \quad \tau \in\{1, \ldots, N-1\} \\
& & \text { (no transmission) }
\end{array}\right.
$$

In (3), when a transmission occurs, which may happen at any time in $\{1, \ldots, N\}, \tau$ is reset to 1 , otherwise $\tau$ is incremented by 1 and this may occur when $\{1, \ldots, N-1\}$, but not when $\tau=N$ as a transmission necessarily needs to be triggered in this case (otherwise the inter-transmission time will be strictly bigger than $N$, which is excluded). When $\tau \in\{1, \ldots, N-1\}$, a transmission may or may not occur. We see that system (3) generates non-unique solutions for a given initial condition: that is not an issue for the forthcoming analysis.

The objective of this paper is to provide conditions to guarantee the stability of the closed-loop system (1)-(2) in presence of communication constraints. In particular, we aim at providing an easily computable bound on $N$. We first need to model the overall system for this purpose.

The control input applied to system (1) is no longer $u$, but a sampled version of input $u$, which we denote $\hat{u}$. Similarly, controller (2) receives the sampled version of $y$, denoted $\hat{y}$. Hence, (1) and (2) respectively become $x_{p}^{+}=f_{p}\left(x_{p}, \hat{u}\right)$ (the output equation does not change) and $x_{c}^{+}=f_{c}\left(x_{c}, \hat{y}\right), u=$ $g_{c}\left(x_{c}, \hat{y}\right)$. The variables $\hat{u}$ and $\hat{y}$ have the following dynamics 
like in [14]

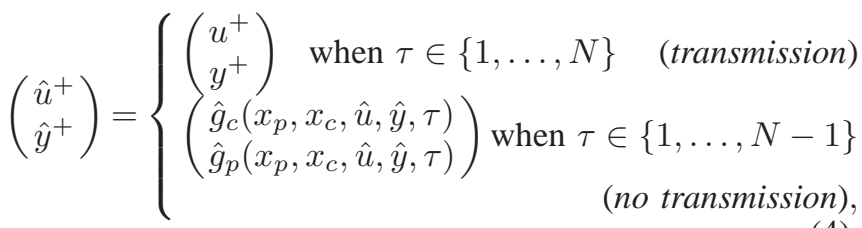

where $y^{+}=g_{p}\left(x_{p}^{+}\right)=g_{p}\left(f_{p}\left(x_{p}, \hat{u}\right)\right)$ and $u^{+}=$ $g_{c}\left(x_{c}^{+}, y^{+}\right)=g_{c}\left(f_{c}\left(x_{c}, \hat{y}\right), g_{p}\left(f_{p}\left(x_{p}, \hat{u}\right)\right)\right)$. The mappings $\hat{g}_{c}$ and $\hat{g}_{p}$ model the way the variables $\hat{u}$ and $\hat{y}$ are respectively generated by the plant and the controller when there is no transmission. A typical implementation is zero-order-hold, meaning that $\hat{u}$ and $\hat{y}$ remain constant in absence of transmission, i.e. $g_{c}\left(x_{p}, x_{c}, \hat{u}, \hat{y}, \tau\right)=\hat{u}$ and $g_{p}\left(x_{p}, x_{c}, \hat{u}, \hat{y}, \tau\right)=\hat{y}$. Another common possibility is to 'zero' $\hat{y}$ and $\hat{u}$ when no transmission occurs [24], which give $g_{c}=0$ and $g_{p}=0$. We allow $\hat{g}_{c}$ and $\hat{g}_{p}$ to depend on all the variables of the problem for the sake of generality, hence covering other types of holding policy, such as the model-based one [16].

For the sake of convenience, and as commonly done in the NCS literature, we introduce the sampling-induced error $e:=\left(e_{u}, e_{y}\right) \in \mathbb{R}^{n_{e}}$ with $e_{u}:=\hat{u}-u, e_{y}:=\hat{y}-y$ and $n_{e}:=n_{u}+n_{y}$. The overall system is modeled as follows

$$
\begin{aligned}
& x^{+}=f(x, e)
\end{aligned}
$$

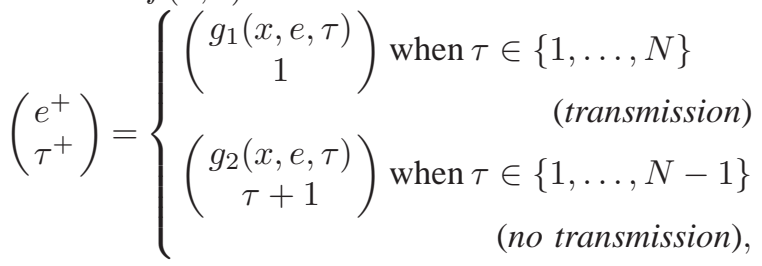

where $x:=\left(x_{c}, x_{p}\right) \in \mathbb{R}^{n_{x}}, n_{x}:=n_{p}+n_{c}, f(x, e):=$ $\left(f_{p}\left(x_{p}, g_{c}\left(x_{c}, g_{p}\left(x_{p}\right)+e_{y}\right)+e_{u}\right), f_{c}\left(x_{c}, g_{p}\left(x_{p}\right)+e_{y}\right)\right)$, $g_{1}(x, e, \tau):=(0,0)$ and $g_{2}(x, e, \tau):=\left(\hat{g}_{c}\left(x, e_{u}+u, e_{y}+\right.\right.$ $y, \tau)-g_{c}\left(f_{c}\left(x_{c}, y+e_{y}\right), \hat{g}_{p}\left(x, e_{u}+u, e_{y}+y, \tau\right)\right), \hat{g}_{p}\left(x, e_{u}+\right.$ $\left.\left.u, e_{y}+y, \tau\right)-g_{p}\left(f_{p}\left(x_{p}, u+e_{u}\right)\right)\right)$, with $u=g_{c}\left(x_{c}, g_{p}\left(x_{p}\right)+\right.$ $\left.e_{y}\right)$ and $y=g_{p}\left(x_{p}\right)$.

\section{MAIN RESULT}

In this section, we first state the assumptions we make on system (5), we then provide a bound on $N$, which we analyse, and we finally state the main stability results.

\section{A. Assumption}

We make the following assumption on system (5).

Assumption 1: There exist $V: \mathbb{R}^{n_{x}} \rightarrow \mathbb{R}_{\geq 0}$ continuous, $W: \mathbb{R}^{n_{e}} \rightarrow \mathbb{R}_{\geq 0}$ continuous, $\underline{\alpha}_{V}, \bar{\alpha}_{V}, \underline{\alpha}_{W}, \bar{\alpha}_{W}, \alpha, \varepsilon \in \mathcal{K}_{\infty}$, $H: \mathbb{R}^{n_{x}} \rightarrow \mathbb{R}$ continuous, $\theta \geq \gamma>0, L \in \mathbb{R}_{\geq 0}$ and $\Delta>0$ such that the following holds.

(i) For any $(x, e) \in \mathbb{R}^{n_{x}+n_{e}}, \underline{\alpha}_{V}(|x|) \leq V(x) \leq \bar{\alpha}_{V}(|x|)$ and $\underline{\alpha}_{W}(|e|) \leq W(e) \leq \bar{\alpha}_{W}(|e|)$

(ii) For any $(x, e) \in \mathbb{R}^{n_{x}+n_{e}}$ such that $\max \{|x|,|e|\} \leq$ $\Delta, V(f(x, e))-V(x) \leq-\alpha(|x|)-\varepsilon(|e|)-\theta H^{2}(x)$ $+\gamma W^{2}(e)$.

(iii) For any $(x, e) \in \mathbb{R}^{n_{e}}$ such that $\max \{|x|,|e|\} \leq \Delta$, $W\left(g_{2}(x, e, \tau)\right) \leq L W(e)+H(x)$.
When items (ii)-(iii) hold for any $(x, e) \in \mathbb{R}^{n_{x}+n_{e}}$, we say that the assumption holds globally.

Item (i) of Assumption 1 means that $V$ and $W$ are positive definite and radially unbounded. Item (ii) of Assumption 1 is a robust local stability property of the system $x^{+}=f(x, e)$. When the loop is closed at each step, $e=0$, and items (i)(ii) imply that the origin is uniformly locally asymptotically stable for the system $x^{+}=f(x, 0)$, which corresponds to the closed-loop system (1)-(2). When $e \neq 0$, these items imply that the $x$-system satisfies an input-to-stable stability property with input $e$, and also that the $x$-system is (locally) $\ell_{2}$-stable from $W(e)$ to $H(x)$ with gain $\sqrt{\frac{\gamma}{\theta}}$. It is interesting to note that this gain is less than 1 since $\gamma \leq \theta$. This is an important difference compared to the continuous-time results in [18] where there is no condition on the corresponding $\mathcal{L}_{2}$-gain. A possible explanation is the following. The underlying idea in [18] is that the $e$-system is $\mathcal{L}_{2}$-stable from $H(x)$ to $W(e)$, with a gain that can be made arbitrarily small, by selecting the MATI bound accordingly (see Proposition 6 in [17] for a formal statement), then a small-gain analysis allows ensuring the stability of the overall system. In our study, we cannot make this $\ell_{2}$-gain as small as desired because the MATI, $N$, is a strictly positive integer. When $\gamma>\theta$, our analysis gives $N=1$, that is we transmit at each step, as $e$ is always equal to 0 . Item (iii) of Assumption 1 is an exponential growth condition of the dynamics of the $e$-system when there is no transmission. When system (1) is linear and time-invariant, the conditions in Assumption 1 can be written as a linear matrix inequality, as explained in Section V-A. An example of a nonlinear system that verifies Assumption 1 is provided in Section V-B.

\section{B. MATI estimate}

To provide a bound on $N$, we introduce the variable $\phi \in$ $\mathbb{R}_{\geq 0}$ which has the following dynamics

$$
\phi^{+}=\lambda \frac{\phi-1}{\phi-1+\lambda L^{2}}, \quad \phi(1)=\lambda,
$$

where $\lambda:=\frac{\theta}{\gamma} \geq 1$, and $\theta, \gamma, L$ come from Assumption 1 . We define the MATI as

$$
N^{\star}:=\sup \left\{k \in \mathbb{Z}_{>0}: \phi(k) \geq 1\right\} .
$$

Note that the set in the right-hand side of (6) is never empty as $\phi(1)=\lambda=\frac{\theta}{\gamma}>1(\theta>\gamma$ according to Assumption 1). Moreover, the denominator in (6) never cancels when $\phi \geq 1$ (unless $L=0$, in which case we define $\phi^{+}=\lambda$ ). We provide guidelines on how to compute $N^{\star}$ below but before that we state the following result, which provides necessary and sufficient conditions under which $N^{\star}$ is respectively (in)finite and strictly bigger than 1 .

Proposition 1: The following holds.

(i) $N^{\star}$ is finite if and only if $L>1-\sqrt{\lambda^{-1}}$.

(ii) $N^{\star} \geq 2$ if and only if $L \leq \sqrt{\lambda}-\sqrt{\lambda}^{-1}$.

Proof. We first prove item (i) of Proposition 1. The idea is to show that $\phi(k)$, as iteratively defined by (3), does not increase. We will see that two situations can then happen: either the iterative map in (3) has a fixed point that belongs 
to $[1, \lambda]$, in which case $\phi$ converges to it as time grows and $N^{\star}$ is infinite, or there is no such fixed point and $\phi$ becomes strictly less than 1 in finite time.

For the sake of convenience, we introduce $\psi:=\phi-1$. To show that $\psi(k)$ is non-increasing is thus equivalent to show that $\phi(k)$ is non-increasing. We have

$$
\psi^{+}=\phi^{+}-1=\lambda \frac{\psi}{\psi+\lambda L^{2}}-1=\frac{(\lambda-1) \psi-\lambda L^{2}}{\psi+\lambda L^{2}} .
$$

Let $\psi \in[0, \lambda-1]$,

$$
\begin{aligned}
\psi^{+} \leq \psi & \Leftrightarrow \quad \frac{(\lambda-1) \psi-\lambda L^{2}}{\psi+\lambda L^{2}} \leq \psi \\
& \Leftrightarrow \quad(\lambda-1) \psi-\lambda L^{2} \leq \psi^{2}+\lambda L^{2} \psi \\
& \Leftrightarrow \quad 0 \leq \psi^{2}+\left(\lambda\left(L^{2}-1\right)+1\right) \psi+\lambda L^{2} .
\end{aligned}
$$

We interpret the last term on the right-hand side above as a second-order polynomial in $\psi$, which we denote $p$. Its discriminant is $\delta:=\left(\lambda\left(L^{2}-1\right)+1\right)^{2}-4 \lambda L^{2}$. When $\delta<0, p$ has no real root, therefore $p(\psi)>0$ for all $\psi$, which means that any solution to (8) strictly decreases and (8) has no fixed point, hence $N^{\star}$ is finite.

Consider now the case where $\delta \geq 0$ and let $X:=\lambda\left(L^{2}-\right.$ $1)+1$. We show that the maximal root of $p, \bar{\psi}:=\frac{-X+\sqrt{\delta}}{2}$, is such that $\bar{\psi}<\lambda-1$. This statement is equivalent to $-X+$ $\sqrt{\delta}=-X+\sqrt{X^{2}-4 \lambda L^{2}}<2(\lambda-1)$, which leads to $-X-2(\lambda-1)<-\sqrt{X^{2}-4 \lambda L^{2}}$. Note that $-X-2(\lambda-1) \leq$ 0 , otherwise we would have $-2(\lambda-1)>X=\lambda\left(L^{2}-1\right)+1$, which gives $0>\lambda L^{2}+\lambda-1$, which is false as $\lambda L^{2} \geq 0$ and $\lambda-1 \geq 0$ by definition of $\lambda$. Thus $-X-2(\lambda-1)<$ $-\sqrt{X^{2}-4 \lambda L^{2}}$ is equivalent to $(-X-2(\lambda-1))^{2}>X^{2}-$ $4 \lambda L^{2}$, that we write as $X^{2}+4(\lambda-1) X+4(\lambda-1)^{2}>$ $X^{2}-4 \lambda L^{2}$. After simplifying both sides of the last inequality, we obtain $(\lambda-1) X+(\lambda-1)^{2}+\lambda L^{2}=(\lambda-1)\left(\lambda\left(L^{2}-\right.\right.$ $1)+1)+(\lambda-1)^{2}+\lambda L^{2}=\lambda^{2} L^{2}>0$ which is true as long as $L>0$. Hence, $\bar{\psi}<\lambda-1$ when $L>0$. For any $\psi>\max \{\bar{\psi}, 0\}, \psi^{+}>\bar{\psi}$ since $\psi>\bar{\psi}$ implies $\psi^{+}>\bar{\psi}^{+}=$ $\bar{\psi}$, as the right-hand side of (8) is strictly increasing in $\psi$ on $\mathbb{R}_{>0}$. Consequently, $\psi(k)$ iteratively defined by (8) and initialized at $\lambda$ strictly decreases when $L>0$. When $L=0$, $\psi(k)=\lambda-1$ for any $k \in \mathbb{Z}_{\geq 0}$ according to (8).

We have thus proved that $\psi(k)$ is non-increasing in all cases. We deduce that $N$ is finite if and only if (8) has no fixed point (i.e. $\delta<0$ ) or when $\bar{\psi}<0$. The first case, i.e. $\delta<0$, is equivalent to $L \in\left(1-\sqrt{\lambda}^{-1}, 1+\sqrt{\lambda}^{-1}\right)$. We next analyse the second case. We have $\bar{\psi}<0$ and $\delta \geq 0$ is equivalent to $-X+\sqrt{X^{2}-4 \lambda L^{2}}<0$ and $X^{2}-4 \lambda L^{2} \geq$ 0 . These inequalities are equivalent to $X \geq 2 \sqrt{\lambda} L$, that is $\lambda\left(L^{2}-1\right)+1 \geq 2 \sqrt{\lambda} L$, which gives $(\sqrt{\lambda} L-1)^{2} \geq \lambda$ and finally $L \geq 1+\sqrt{\lambda}^{-1}$. As a result, $N^{\star}$ is finite if and only if $L>1-\sqrt{\lambda}^{-1}$.

To prove item (ii) of Proposition 1, we study when $\phi(2) \geq$ 1 with $\phi(1)=\lambda$, that is when $\lambda \frac{\lambda-1}{\lambda-1+\lambda L^{2}} \geq 1$. The latter is equivalent to $\lambda(\lambda-1) \geq \lambda-1+\lambda L^{2}$, which we rewrite as $\lambda^{2}-2 \lambda+1=(\lambda-1)^{2} \geq \lambda L^{2}$. Hence $\phi(2) \geq 1$ if and only if $\lambda-1 \geq \sqrt{\lambda} L$, which corresponds to the condition in item (ii) of Proposition 1.

According to Proposition 1, when $L>1-\sqrt{\lambda^{-1}}, N^{\star}$ is finite. To compute it, we can run a simple program where we initialize $\phi$ at $\lambda$, and we iterate it according to (6) until it becomes strictly less than 1 at iteration $N^{\star}+1$. When, in addition, $L \geq \sqrt{\lambda}-\sqrt{\lambda}^{-1}$, we immediately know that $N^{\star}=1$ according to item (ii) of Proposition 1, which means that we transmit at each step. When $L<1-\sqrt{\lambda^{-1}}, N^{\star}$ is infinite, which means that we only need to close the feedback loop once. This situation may happen in specific scenarios, like when $y=x$ and model-based holding functions are to generate $\hat{u}$ and $\hat{x}$, or when controller (2) stabilizes the origin of system (1) in one step and the zero-input holding strategy is employed.

Remark 1: In [18], where the plant and the controller have continuous-time dynamics, a similar variable $\phi$ is introduced to compute the MATI. In particular, $\phi$ is given by the solution to a nonlinear ordinary differential equation (see (27) in [18]). The latter is solved analytically and then the MATI bound is obtained. To analytically determine $N^{\star}$ is a difficult task, which is the reason why we have decided to define it as in (7), which is easy to evaluate as explained above.

\section{Stability guarantees}

The next result ensures asymptotic stability properties for system (5).

Theorem 1: Consider system (5) and suppose Assumption 1 holds. For any $N \in\left\{1, \ldots, N^{\star}\right\}$, where $N^{\star}$ is defined in (7), the compact set $\mathcal{A}:=\{(x, e, \tau): x=0, e=0, \tau \in\{1, \ldots, N\}\}$ is:

(i) ULAS;

(ii) UGAS when Assumption 1 holds globally;

(iii) ULES (respectively, UGES) when Assumption 1 holds (respectively, globally) with $\underline{\alpha}_{V}(s)=\underline{a}_{V} s^{2}, \bar{\alpha}_{V}(s)=$ $\bar{a}_{V} s^{2}, \underline{\alpha}_{W}(s)=\underline{a}_{W} s^{2}, \bar{\alpha}_{W}(s)=\bar{a}_{W} s^{2}, \alpha(s)=a s^{2}$, $\varepsilon(s)=\epsilon s^{2}$ for any $s \geq 0$, with $\underline{a}_{V}, \bar{a}_{V}, \underline{a}_{W}, \bar{a}_{W}, a, \epsilon>$ 0 .

Proof. We prove item (i) of Theorem 1, the two other items similarly follow. We write system (5) as $q^{+} \in F(q)$ with $q:=(x, e, \tau)$ for the sake of convenience. We introduce $U(q)=V(x)+\gamma \phi(\tau) W^{2}(e)$ for any $(x, e) \in \mathbb{R}^{n_{x}+n_{e}}$ and $\tau \in\{1, \ldots, N\}$, where $V, W, \gamma$ come from Assumption 1. Let $(x, e) \in \mathbb{R}^{n_{x}+n_{e}}$ be such that $\max \{|x|,|e|\} \leq \Delta$, where $\Delta$ comes from Assumption 1 , and $\tau \in\{1, \ldots, N\}$. Let $\varphi \in F(q)$. From item (ii) of Assumption 1, when $\varphi=\left(f(x, e), g_{1}(x, e, \tau), 1\right)$, i.e. when a transmission occurs,

$$
\begin{aligned}
U(\varphi)-U(q) \leq & -\alpha(|x|)-\varepsilon(|e|)-\theta H^{2}(x)+\gamma W^{2}(e) \\
& +\gamma \phi(1) W^{2}(0)-\gamma \phi(\tau) W^{2}(e) \\
= & -\alpha(|x|)-\varepsilon(|e|)-\theta H^{2}(x)+\gamma W^{2}(e) \\
& -\gamma \phi(\tau) W^{2}(e)
\end{aligned}
$$

By definition of $N^{\star}$ in (7) and since $\tau \leq N \leq N^{\star}, \phi(\tau) \geq 1$, thus $\gamma W^{2}(e)-\gamma \phi(\tau) W^{2}(e) \leq 0$ and

$$
U(\varphi)-U(q) \leq-\alpha(|x|)-\varepsilon(|e|) \leq-\rho(|(x, e)|),
$$


for some $\rho \in \mathcal{K}_{\infty}$.

When $\varphi=\left(f(x, e), g_{2}(x, e, \tau), \tau+1\right)$, i.e. when no transmission occurs, $\tau \in\{1, \ldots, N-1\}$ and in view of items (ii)-(iii) of Assumption 1,

$$
\begin{aligned}
& U(\varphi)-U(q) \leq-\alpha(|x|)-\varepsilon(|e|)-\theta H^{2}(x)+\gamma W^{2}(e) \\
& \quad+\gamma \phi(\tau+1) W^{2}\left(g_{2}(x, e, \tau)\right)-\gamma \phi(\tau) W^{2}(e) \\
& \leq-\alpha(|x|)-\varepsilon(|e|)-\theta H^{2}(x)+\gamma W^{2}(e) \\
&+\gamma \phi(\tau+1)(L W(e)+H(x))^{2}-\gamma \phi(\tau) W^{2}(e) \\
&=-\alpha(|x|)-\varepsilon(|e|)-\theta H^{2}(x)+\gamma W^{2}(e) \\
&+\gamma \phi(\tau+1)\left(L^{2} W(e)^{2}+H(x)^{2}+2 L W(e) H(x)\right) \\
&-\gamma \phi(\tau) W^{2}(e) \\
&=-\alpha(|x|)-\varepsilon(|e|) \\
&-\langle(H(x), W(e)), \mathcal{M}(\tau)(H(x), W(e))\rangle,
\end{aligned}
$$

where

$$
\mathcal{M}(\tau):=\left(\begin{array}{cc}
\theta-\gamma \phi(\tau+1) & -\gamma \phi(\tau+1) L \\
\star & \gamma\left(-1+\phi(\tau)-\phi(\tau+1) L^{2}\right)
\end{array}\right) .
$$

The matrix $\mathcal{M}(\tau)$ is positive semi-definite if and only if

$$
\left\{\begin{aligned}
0 \leq & \theta-\gamma \phi(\tau+1) \\
0 \leq & (\theta-\gamma \phi(\tau+1)) \gamma\left(-1+\phi(\tau)-\phi(\tau+1) L^{2}\right) \\
& -\gamma^{2} \phi(\tau+1)^{2} L^{2} .
\end{aligned}\right.
$$

The first inequality follows from the fact that $\phi(\tau) \leq \frac{\theta}{\gamma}$ for all $\tau \in\{1, \ldots, N\}$, as $\phi$ is shown to be non-increasing in the proof of Proposition 1 and $\phi(1)=\frac{\theta}{\gamma}$. The definition of $\phi(\tau+1)$ in (6) is such that the left-hand side of the second inequality above cancels. Indeed, the latter can be written as $\left(-\gamma^{2}(-1+\phi(\tau))-\gamma \theta L^{2}\right) \phi(\tau+1)+\theta \gamma(-1+$ $\phi(\tau)$ ) (the terms in $\phi(\tau+1)^{2}$ cancel), whose root, when interpreting $\phi(\tau+1)$ as an unknown, is $\phi(\tau+1)=$ $\frac{\theta \gamma(1-\phi(\tau))}{-\gamma^{2}(-1+\phi(\tau))-\gamma L^{2} \theta}=\frac{\theta}{\gamma} \frac{\phi(\tau)-1}{\phi(\tau)-1+L^{2} \frac{\theta}{\gamma}}$, which corresponds to (6). The matrix $\mathcal{M}(\tau)$ is therefore positive semi-definite, hence $U(\varphi)-U(q) \leq-\alpha(|x|)-\varepsilon(|e|) \leq$ $-\rho(|(x, e)|)$ with $\rho$ as in $(11)$.

We have proved that $U(\varphi)-U(q) \leq-\rho(|(x, e)|)$ for any $\varphi \in F(q)$. Let $\bar{\Delta}=\underline{\alpha}_{U}(\Delta)$ where $\underline{\alpha}_{U}(s)=$ $\min \left\{\underline{\alpha}_{V}\left(\frac{s}{2}\right), \gamma \underline{\alpha}_{W}^{2}\left(\frac{s}{2}\right)\right\}$ for $s \geq 0$ (see for instance Lemma 2 in [22]), $U(q) \leq \bar{\Delta}$ implies $\max \{|x|,|e|\} \leq \Delta$ in view of item (i) of Assumption 1. The set $\{q: U(q) \leq \bar{\Delta}, \tau \in$ $\{1, \ldots, N\}\}$ is thus forward invariant for system (5). We note that $\underline{\alpha}_{U}\left(|q|_{\mathcal{A}}\right) \leq U(q) \leq \bar{\alpha}_{U}\left(|q|_{\mathcal{A}}\right)$ where $\bar{\alpha}_{U}: s \mapsto$ $\bar{\alpha}_{V}(s)+\theta \bar{\alpha}_{W}(s) \in \mathcal{K}_{\infty}$ and $\underline{\alpha}_{U} \in \mathcal{K}_{\infty}$. We deduce that the set $\mathcal{A}$ is ULAS using Theorem 8 in [20].

Remark 2: The stability properties ensured by Theorem 1 are robust to the so-called $\sigma$ - or $\rho$-perturbations when $f(x, e)$, $g_{1}(x, e, \tau), g_{2}(x, e, \tau)$ in (5) are compact and non-empty for any $x, e, \tau$ (which is the case when the corresponding mappings are continuous for instance), according to Theorem 2.8 in [12] as the Lyapunov function used in the proof of Theorem 1 is continuous.

\section{Applications}

In this section, we apply the results of the previous section to linear time-invariant systems and to a nonlinear example.

\section{A. Linear time-invariant systems}

Consider the system

$$
x_{p}^{+}=A_{p} x_{p}+B_{p} u, \quad y=C_{p} x_{p},
$$

where $\left(A_{p}, B_{p}\right)$ is stabilizable and $\left(A_{p}, C_{p}\right)$ is detectable. We can therefore stabilize the origin of system (15) using a dynamic controller of the form

$$
x_{c}^{+}=A_{c} x_{c}+B_{c} y, \quad u=C_{c} x_{c}+D_{c} y .
$$

We take into account the communication constraints between system (15) and controller (16), and we obtain the model below in view of Section III

$$
\begin{aligned}
x^{+} & =\mathcal{A}_{1} x+\mathcal{B}_{1} e \\
\left(\begin{array}{c}
e^{+} \\
\tau^{+}
\end{array}\right) & =\left\{\begin{array}{rr}
\left(\begin{array}{c}
(0,0) \\
1
\end{array}\right) & \text { when } \tau \in\{1, \ldots, N\} \\
\left(\begin{array}{c}
\mathcal{A}_{2} x+\mathcal{B}_{2} e \\
\tau+1
\end{array}\right) \text { when } \tau & \in\left\{\begin{array}{r}
\text { (transmission) } \\
1, \ldots, N-1\}
\end{array}\right. \\
& \text { (no transmission) },
\end{array}\right.
\end{aligned}
$$

where $x=\left(x_{c}, x_{p}\right) \in \mathbb{R}^{n_{x}}$, and $\mathcal{A}_{1}:=$ $\left(\begin{array}{cc}A_{p}+B_{p} D_{c} C_{p} & B_{p} C_{p} \\ B_{p} C_{p} & A_{c}\end{array}\right), \mathcal{B}_{1}:=\left(\begin{array}{cc}B_{p} D_{c} & B_{p} \\ B_{c} & 0\end{array}\right)$, $\mathcal{A}_{2}:=\left(\begin{array}{cc}-C_{p}\left(A_{p}+B_{p} D_{c} C_{p}\right) & -C_{p} B_{p} C_{p} \\ -C_{c} B_{c} C_{p} & -C_{c} A_{c}\end{array}\right), \quad$ and $\mathcal{B}_{2}:=\left(\begin{array}{cc}-C_{p} B_{p} D_{c} & -C_{p} B_{p} \\ -C_{c} B_{c} & 0\end{array}\right)$.

The next result shows that system (15) ensures Assumption 1 provided a linear matrix inequality holds, which then implies that the set $\mathcal{A}$ is UGES according to Theorem 1. Its proof is omitted for space reasons.

Proposition 2: Consider system (17) and suppose that there exists a symmetric positive definite matrix $P, \theta \geq \gamma>$ 0 such that

$$
\left(\begin{array}{cc}
\mathcal{A}_{1}^{\mathrm{T}} P \mathcal{A}_{1}-P+\theta \mathcal{A}_{2}^{\mathrm{T}} \mathcal{A}_{2} & \mathcal{A}_{1}^{\mathrm{T}} P \mathcal{B}_{1} \\
\star & -\gamma \mathbb{I}+\mathcal{B}_{1}^{\mathrm{T}} P \mathcal{B}_{1}
\end{array}\right)<0 .
$$

Then, Assumption 1 holds globally and the set $\mathcal{A}$ defined in Theorem 1 is UGES when $N \leq N^{\star}$ with $N^{\star}$ given by (7).

\section{B. Lorenz model}

We consider the Euler discretization of the controlled Lorenz model of a thermal convection loop ${ }^{1}$ studied in [26] with sampling period $T>0$

$$
\begin{aligned}
& x_{1}^{+}=x_{1}+T\left(-a x_{1}+a x_{2}\right) \\
& x_{2}^{+}=x_{2}+T\left(b x_{1}-x_{2}-x_{1} x_{3}+u\right) \\
& x_{3}^{+}=x_{3}+T\left(x_{1} x_{2}-c x_{3}\right) \\
& y=x_{1},
\end{aligned}
$$

where $a, b, c>0$, see [26] for details on the meaning of the state variables, the control input and the parameters. We take $a=10, b=28, c=\frac{8}{3}$ and $T=10^{-3}$. We design the static

\footnotetext{
${ }^{1}$ A careful analysis of the relationship between the stability of this model and the stability of the original sampled-data one would be interesting but is outside the scope of this paper, see [19].
} 
output-feedback law $u=-b y$. After taking into account the communication constraints, the input applied to the plant is $u=-b(y+e)$ where $e=\hat{y}-y$ (there is no need to introduce the error on $u$ since the controller is static).When using the hold-input strategy, the corresponding system satisfies Assumption 1 with $W(e)=|e|, \underline{\alpha}_{W}(s)=\bar{\alpha}_{W}(s)=s$, $L=1, H(x)=T\left|-a x_{1}+a x_{2}\right|, V(x)=0.0059288 x_{1}^{4}+$ $2.8058 \cdot 10^{-6} x_{2}^{4}+0.0044086 x_{3}^{4}+2.5782 x_{1}^{2}-4.9865 x_{1} x_{2}+$ $8.0907 \cdot 10^{-7} x_{1} x_{3}+5.3294 x_{2}^{2}-7.4358 \cdot 10^{-7} x_{2} x_{3}+3.0477 x_{3}^{2}$, which was obtained using SOSTOOLS [21], $\underline{\alpha}_{V}(s)=s^{2}$ and some $\bar{\alpha} \in \mathcal{K}_{\infty}, \alpha(s)=\varepsilon(s)=10^{-3} s^{2}, \theta=200, \gamma=2.001$, and $\Delta=\sqrt{1000}$, for $x=\left(x_{1}, x_{2}, x_{3}\right) \in \mathbb{R}^{3}, e \in \mathbb{R}$ and $s \geq 0$. In particular, item (ii) of Assumption 1 holds for any $|x| \leq \Delta$ and $e \in \mathbb{R}$, and item (iii) of Assumption 1 is verified for any $x, e$. As a consequence, the set $\mathcal{A}$ defined in Theorem 1 is ULAS. Furthermore, in this case, the set $\left\{(x, e, \tau): V(x)+\gamma \phi(\tau) W^{2}(e) \leq \Delta^{2}\right\}$ belongs to the basin of attraction of the origin (since $V(x) \geq|x|^{2}$ for any $x \in \mathbb{R}^{3}$ ).

The formula in (7) gives $N^{\star}=18$, while simulation results of periodic transmissions have shown that the asymptotic stability of the set $\mathcal{A}$ for the closed-loop system is preserved up to $N^{\star}=77$. The bound we have obtained can be further improved by taking 'smaller' functions $\varepsilon$ and $\alpha$, nevertheless this may affect the robustness of the closedloop system. Also, a different Lyapunov function would generally lead to a different $N^{\star}$ (as well as a different basin of attraction). Finally, we note that Assumption 1 does not hold when 'zeroing' $\hat{y}$ when no transmission occurs (i.e. when $g_{2}=0$ in (5)). In this case, item (iii) of Assumption 1 is verified with $L=0$ (and $H(x)=\left|x_{1}+T\left(-a x_{1}+a x_{2}\right)\right|$ ) but item (ii) of Assumption 1 cannot be satisfied, otherwise $N^{*}$ would be infinite according to item (i) of Proposition 1 (as $L=0$ ), which means that the origin of the open-loop system would be locally exponentially stable, which is not the case.

\section{Conclusions}

We have investigated the scenario in which a discrete-time controller and a discrete-time plant communicate with each other at least every $N$ steps. Assuming that the corresponding closed-loop system satisfies a robust asymptotic stability property when there is no communication constraint, we have provided an explicit bound on $N$ to preserve stability. The results have been applied to linear time-invariant systems in which case the assumptions are written as a linear matrix inequality, as well as to a nonlinear physical example.

This study was motivated by [25], where we develop energy-efficient transmissions strategy for time-triggered controlled discrete-time systems implemented over a wireless network.

\section{ACKNOWLEDGEMENT}

Romain Postoyan would like to thank Vineeth S. Varma for the discussions which lead to this work, and Giorgio Valmorbida for his precious advices regarding the use of SOSTOOLS in Section V-B.

\section{REFERENCES}

[1] T. Bastogne and J.-B. Tylcz. Biomedical device to control the activation of a photosensitizing agent in a biological tissue, Patent number: 13193028.1-1652, 2013.

[2] A. Bemporad. Predictive control of teleoperated constrained systems with unbounded communication delays. In IEEE Conf. on Decision and Control, Tampa, U.S.A., volume 2, pages 2133-2138, 1998.

[3] L. Buşoniu, R. Postoyan, and J. Daafouz. Near-optimal strategies for nonlinear and uncertain networked control systems. IEEE Transactions on Automatic Control, 61(8):2124-2139, 2016.

[4] A. Chaillet and A. Bicchi. Delay compensation in packet-switching networked controlled systems. In IEEE Conference on Decision and Control, Cancun, Mexico, pages 3620-3625, 2008.

[5] R. Cogill. Event-based control using quadratic approximate value functions. In IEEE Conference on Decision and Control, Shanghai, China, pages 5883-5888, 2009.

[6] M.C.F. Donkers, W.P.M.H. Heemels, N. van de Wouw, and L. Hetel. Stability analysis of networked control systems using a switched linear systems approach. IEEE Transactions on Automatic Control, 56(9):2101-2115, 2011.

[7] T.J. Dougherty, C.J. Gomer, B.W. Henderson, G. Jori, D. Kessel, M. Korbelik, J. Moan, and Q. Peng. Photodynamic therapy. Journal of the National Cancer Institute, 90(12):889-905, 1998.

[8] A. Eqtami, V. Dimarogonas, and K. Kyriakopoulos. Event-triggered control for discrete-time systems. In American Control Conference, Baltimore, U.S.A., pages 4719-4724, 2010.

[9] T.M.P. Gommans and W.P.M.H. Heemels. Resource-aware MPC for constrained nonlinear systems: a self-triggered control approach. Systems \& Control Letters, 79:59-67, 2015.

[10] W.P.M.H. Heemels, K.H. Johansson, and P. Tabuada. An introduction to event-triggered and self-triggered control. In IEEE Conference on Decision and Control, Maui, U.S.A., pages 3270-3285, 2012.

[11] W. Hu, G. Liu, and D. Rees. Event-driven networked predictive control. IEEE Trans. on Ind. Electronics, 54(3):1603-1613, 2007.

[12] C.M. Kellett and A.R. Teel. On the robustness of $\mathcal{K} \mathcal{L}$-stability for difference inclusions: smooth discrete-time Lyapunov functions. SIAM Journal on Control and Optimization, 44(3):777-800, 2005.

[13] $\mathrm{L}$. $\mathrm{Li}$ and $\mathrm{M}$. Lemmon. Weakly coupled event triggered output feedback system in wireless networked control systems. Discrete Event Dynamic Systems, 24(2):247-260, 2014.

[14] M. Lješnjanin, D.E. Quevedo, and D. Nešić. Controllability of discrete-time networked control systems with try once discard protocol. In IFAC World Congress, Cape Town, South Africa, pages 37583763, 2014.

[15] L. Ljung. System identification. Prentice Hall, Englewood Cliffs, New Jersey, U.S.A., 1987.

[16] J. Lunze and D. Lehmann. A state-feedback approach to event-based control. Automatica, 46:211-215, 2010.

[17] D. Nešić and A.R. Teel. Input-output stability properties of networked control systems. IEEE Trans. on Aut. Control, 49:1650-1667, 2004.

[18] D. Nešić, A.R. Teel, and D. Carnevale. Explicit computation of the sampling period in emulation of controllers for nonlinear sampled-data systems. IEEE Trans. on Automatic Control, 54(3):619-624, 2009.

[19] D. Nešić, A.R. Teel, and P.V. Kokotović. Sufficient conditions for stabilization of sampled-data systems via discrete-time approximations. Systems \& Control Letters, 38:259-270, 1999.

[20] D. Nešić, A.R. Teel, and E.D. Sontag. Formulas relating $\mathcal{K} \mathcal{L}$ stability estimates of discrete-time and sampled-data nonlinear systems. Systems \& Control Letters, 38(1):49-60, 1999.

[21] A. Papachristodoulou, J. Anderson, G. Valmorbida, S. Prajna, P. Seiler, and P. A. Parrilo. SOSTOOLS: Sum of squares optimization toolbox for MATLAB. http://arxiv.org/abs/1310.4716, 2013.

[22] R. Postoyan, P. Tabuada, D. Nešić, and A. Anta. A framework for the event-triggered stabilization of nonlinear systems. IEEE Transactions on Automatic Control, 60(4):982-996, 2015.

[23] D.E. Quevedo and D. Nešić. Robust stability of packetized predictive control of nonlinear systems with disturbances and Markovian packet losses. Automatica, 48(8):1803-1811, 2012.

[24] L. Schenato. To zero or to hold control inputs with lossy links? IEEE Transactions on Automatic Control, 54(5):1093-1099, 2009.

[25] V.S. Varma and R. Postoyan. Energy efficient time-triggered control over wireless sensor/actuator networks. In IEEE Conference on Decision and Control, Las Vegas, U.S.A., 2016.

[26] J.-C. Wan and D.S. Bernstein. Nonlinear feedback control with global stabilization. Dynamics and Control, 5(4):321-346, 1995. 